\title{
Copper Staining/Labeling and Scanning Electrochemical Microscopy Readout of Proteins on Poly(vinylidene difluoride) Membranes
}

\author{
Maurizio Carano§a, Niels Lion, and Hubert H. Girault* \\ §CS Poster Prize Winner
}

\begin{abstract}
Scanning Electrochemical Microscopy (SECM) is used in combination with copper labeling to visualize proteins on surfaces. Proteins are adsorbed on a poly(vinylidene difluoride) (PVDF) membrane and stained using a standard protocol involving copper salts. The latter are then reduced to copper metal and further detected by SECM with ferrocene methanol as a redox mediator in aqueous solution. During the SECM scan, the potential is held at a value at which the oxidation of the redox mediator occurs and a positive feedback current is detected when scanning over copper clusters. A negative feedback is observed elsewhere.
\end{abstract}

Keywords: Copper staining · Membrane · Poly(vinylidene difluoride) · Protein detection ·

Scanning Electrochemical Microscopy (SECM)

\section{Introduction}

Detecting protein spots on flat surfaces or pseudo two-dimensional surfaces such as gels or polymeric membranes is one of the routine tasks in analytical biochemistry. This is usually accomplished by direct staining techniques, whether with colorimetric or fluorescent detection. Detection of proteins in gels or on membranes can be accomplished by using copper staining both on membranes and native gels [1][2]. Other methods include the use of colloidal gold or silver that can be used as direct staining agents [3]. Fast and sensitive copper staining of proteins resolved by sodium dodecyl sulphate-polyacrylamide gel electrophoresis was found not to interfere with the sub-

${ }^{*}$ Correspondence: Prof. H.H. Girault

Laboratoire d'Electrochimie Physique et Analytique (LEPA)

Ecole Polytechnique Fédérale de Lausanne (EPFL)

$\mathrm{CH}-1015$ Lausanne

Tel.: +41216933151

Fax: +41216933667

E-Mail: hubert.girault@epfl.ch

aPresent address: Georgia Institute of Technology

Environmental Science \& Technology (ES\&T)

331 Ferst Drive

Atlanta, 30332 sequent electrotransfer of the proteins to a solid support [1][4].

Since Scanning Electrochemical Microscopy (SECM) [5] can be used to dissolve copper [6] in solution, the combination of copper-staining techniques and SECM has the potential for improving the classic visualization of protein on membranes. We investigate herein the detection of protein spots on poly(vinylidene difluoride) (PVDF) membranes by SECM with copper staining of proteins, reduction of the copper salts and further dissolution of these copper clusters by the SECM tip with ferrocene/ferrocenium methanol as mediator.

\section{Experimental}

\section{Chemicals and Materials}

All chemicals were used as received. $\mathrm{CuCl}_{2}, \mathrm{NaBH}_{4}$, ferrocene methanol, and $\mathrm{KNO}_{3}$ were purchased from Aldrich and water was purified using a model Milli Q plus 185 from Millipore (conductivity of 18.2 microS $\left.\mathrm{cm}^{-1}\right)$. Bovine serum albumin (66400 Da, $\mathrm{p} I \sim 4.5$ ) was purchased from Sigma (minimum purity 98\%). The PVDF membranes (Immun-Blot ${ }^{\mathrm{TM}}$, for protein blotting, $0.2 \mu \mathrm{m}$ pore size) were purchased from Bio-Rad and cut into circles to fit the electrochemical cell. Alumina 0.3 and 0.05 $\mu \mathrm{m}$ from Buehler, was used to polish all the metal electrodes and Mastertex polishing cloths from Buehler were employed for the SECM disk tip (working electrode).

\section{Copper Staining Procedure}

The procedure followed for the staining process was that introduced by Lee $e t$ al. [4]. A 0.3 M copper chloride solution was prepared using purified water and added to the protein spots and allowed to react for about $30 \mathrm{~min}$. After the sample was dried and washed with abundant water, a 0.3 $\mathrm{M} \mathrm{NaBH}_{4}$ solution was added in order to reduce the copper. After about $5 \mathrm{~min}$ the solution was removed and the sample was carefully rinsed with purified water.

\section{Instrumentation}

CHI 900 SECM equipment was used for all the electrochemical measurements; a Leitz microscope, model Laborlux D and a two megapixel digital camera were used to capture pictures of the samples before the experiments. For the SECM measurements a custom-made electrochemical cell was employed. The cell was fabricated with the purpose to allow a firm and easy positioning of the PVDF membranes. All measurements were performed using a three electrodes set-up: working electrode was a $25 \mu \mathrm{m}$ in diameter platinum disk SECM tip (purchased from $\mathrm{CHI}, \mathrm{RG}$ value was 5), reference electrode was an $\mathrm{Ag} / \mathrm{AgCl}$ or 
a silver wire coil (quasi reference) and the counter electrode was a platinum wire. The microelectrode electrochemical behavior was checked as previously described [7] to make sure that the electrochemical response was the one expected. In particular cyclic voltammetry experiments were carried out along with negative and positive approach curves that were acquired and fitted to theory.

\section{Sample Preparation}

PVDF membranes were first wetted in methanol and then rinsed with Millipore purified water. The samples were prepared by the drop spot technique: $1 \mu \mathrm{l}$ of BSA in pure water at concentrations of $1.5 \mu \mathrm{M}, 15$ and $1.5 \mathrm{nM}$ were spotted on the membrane, corresponding to final amounts of $0.1 \mu \mathrm{g}, 1 \mathrm{ng}$, and $0.1 \mathrm{ng}$ of protein respectively, and a spot surface of $c a .1 \mathrm{~mm}^{2}$. The staining solution was then added to the protein drops already present on the membrane and 30-60 min at least were allowed for the binding process (formation of $\mathrm{Cu}^{2+}$-protein complex). After evaporation of the solvent the membrane was washed with copious amount of deionized water to wash away all the remaining salts. A reducing $\mathrm{NaBH}_{4}$ solution was then added in order to reduce $\mathrm{Cu}^{2+}$ to $\mathrm{Cu}^{0}$. Evaporation of the solvent was again followed by the washing procedure. After checking the status of the samples under the light microscope, the membrane was placed in the SECM cell. A water solution of ferrocene methanol $(1 \mathrm{mM})$ and $\mathrm{KNO}_{3}(0.1 \mathrm{M})$ was used to perform the electrochemical experiments. The strong binding of the protein to the PVDF membrane assured that no relevant amount of sample was dissolved in the electrochemical cell. Approach curves were obtained far from the spots and negative feedback was observed.

\section{Results and Discussion}

Given that the main goal of this study was to explore the possibility of detecting protein spots on PVDF membranes by SECM, bovine serum albumin was used as a model compound. PVDF membranes bind peptides and proteins through hydrophobic interactions with a high binding capacity $\left(140-150 \mu \mathrm{g} / \mathrm{cm}^{2}\right.$ of frontal surface for BSA, supplier data [8]). It is not completely clear whether proteins retain their three-dimensional structure upon capture on the membrane, whereas they conserve their immuno-reactivity in classical Western blot assays where proteins separated by gel electrophoresis are transferred to a PVDF membrane and exposed to specific antibodies. The relatively small spot size (ca. $1 \mathrm{~mm}^{2}$, corresponding to $1.5 \times 10^{-12}$, $15 \times 10^{-14}$ and $1.5 \times 10^{-14} \mathrm{~mol} / \mathrm{mm}^{2}$ ) was chosen to be comparable to spot sizes usu- ally encountered in gel electrophoresis and electroblotting assays ( $c a .1-5 \mathrm{~mm}^{2}$ ). The Scheme shows the principle of the proposed approach to the detection of proteins. The $25 \mu \mathrm{m}$ working electrode is held at +0.25 $\mathrm{V}$ vs $\mathrm{Ag} / \mathrm{AgCl}$; at this value the oxidation of the redox mediator is observed. When the tip is close enough to the sample, the oxidized form of the mediator reacts with the silver nanoparticles leading to the pro-

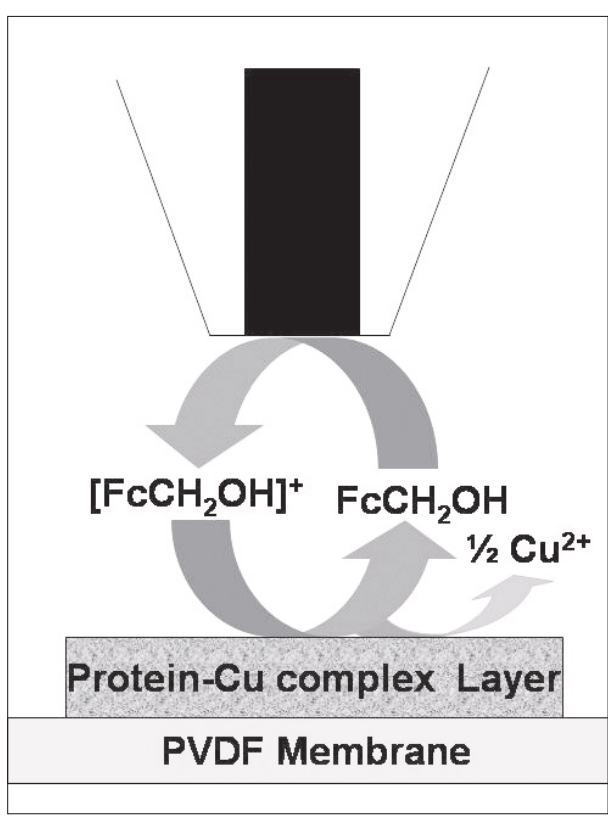

Scheme. Schematic representation of the electrochemical principle used to detect protein through copper dissolution. Dimensions are not to scale. duction of $\mathrm{Cu}^{2+}$. Ferrocene methanol was chosen as mediator because the standard redox potential for the couple ferrocene methanol $^{1+/ 0}(0.480 \mathrm{~V}$ vs SCE [9]) is positive enough that its oxidized form is able to accept electrons from $\mathrm{Cu}$ atoms attached to the protein on the substrate $\left(\mathrm{Cu}^{2+} / \mathrm{Cu}\right.$ standard potential is $0.340 \mathrm{~V} v s$ SCE [10]). Furthermore this potential is not too close to the positive limit of the potential window in water. After proper characterization of the tip used for the experiments, the electrochemical set up was tested by performing cyclic voltammograms $(\mathrm{CV})$ in a $1 \mathrm{mM}$ ferrocene methanol aqueous solution $(0.1 \mathrm{M}$ $\mathrm{KNO}_{3}$ as supporting electrolyte).

Approach curves to the PVDF substrate yielded a negative feedback due to the nonconductive nature of the surface and the absence of non-specific adsorption of copper on bare PVDF. Fig. 1 shows one of these approach curves (solid line) compared to the theoretical values (dotted line). Comparison of the experimental results with the theory revealed that it was possible to place the tip very close to the surface $(2-3 \mu \mathrm{m}$ or less). Nevertheless the fit was not as good as during the characterization of the tip. This could be due to the porosity of the substrate that allows a higher flux of redox species to the electrode up to a closer tip/substrate distance compared to a non-porous material. In this sense, the experimental current is found above that predicted by theory and it decreases more sharply than the latter at very close distances from the substrate. This

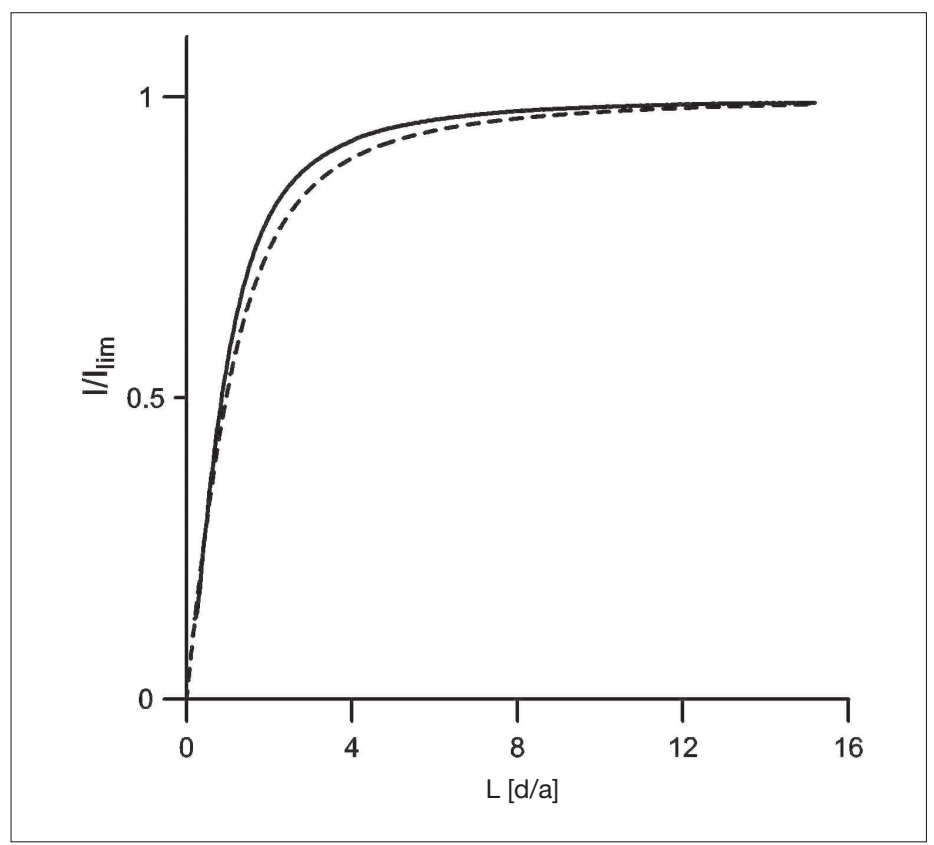

Fig. 1. Scanning approach curve acquired at $2 \mu \mathrm{m} / \mathrm{s}, 25 \mu \mathrm{m}$ platinum disk working electrode, Ag wire as quasi reference electrode, Pt wire as counter electrode. Approach curve is presented in $\mathrm{L}=\mathrm{d} /$ a versus normalized current $\mathrm{I}=\mathrm{i} / \mathrm{i}_{\mathrm{t}, \infty}$ where $\mathrm{d}$ is the traveling distance from the substrate, a the tip radius, $\mathrm{i}$ the recorded current and $\mathrm{i}_{\mathrm{t}, \infty}$ the limiting current. Equilibration time before scan: $50 \mathrm{~s}$ at $0.35 \mathrm{~V} v s \mathrm{Ag}$ wire. $1 \mathrm{mM}$ ferrocene methanol aqueous solution, $0.1 \mathrm{M} \mathrm{KNO}_{3}$ as supporting electrolyte. $\mathrm{T}=25^{\circ} \mathrm{C}$. Dotted line is the simulated curve. 


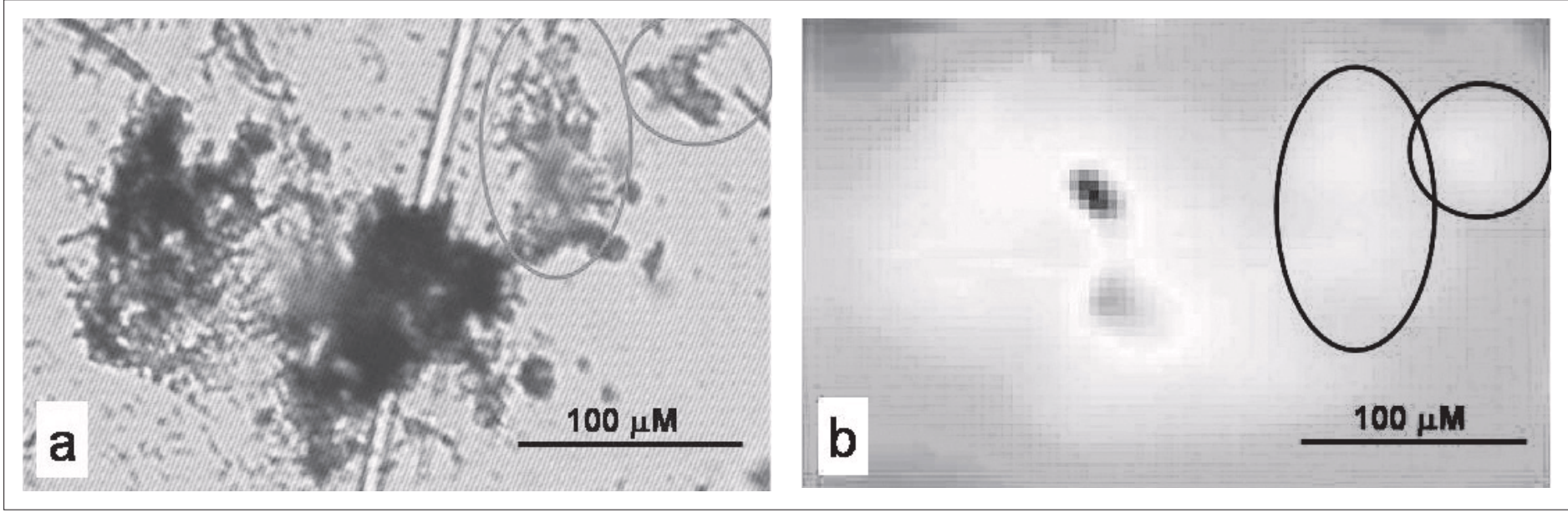

Fig. 2. (a) Optical image of a protein spot ( $1.5 \mathrm{nM}$ protein solution spotted, corresponding to $0.1 \mathrm{ng}$ deposited) after staining with copper and following reduction of copper salt. The visible line was a scratch present on the membrane but it is not visible in the SECM scan because the tip is too far away from the surface. (b) SECM image of the same protein spot. All conditions as in Fig. 1.

means that the current level will be higher in our case at comparable distances. After approaching the membrane, the electrode was withdrawn by about 20 microns. Cyclic voltammetry curves were acquired both before and after the approach scans to check that the feedback regime was preserved. The use of the custom-made electrochemical cell resulted in a very good flatness of the PVDF membrane: a deviation in the range of $1-5 \mu \mathrm{m}$ over $1 \mathrm{~mm}$ scans within several experiments was measured by fitting approach curves to the theory at different locations.

After checking the overall detection system, protein spots were imaged. Fig.2a shows an optical image of a protein spot obtained from a $1.5 \mathrm{nM}$ BSA solution (corresponding to approximately $0.1 \mathrm{ng}$ of protein deposited) after staining with copper and washing with millipore water. As unstained protein spots are not visible on the membrane, all the morphology seen can be attributed to copper staining. Fig. $2 b$ shows the same protein spot imaged by SECM. Though the images are somehow tilted due to different membrane positioning under the microscope or the SECM, the spot shapes are very similar. Two areas that can be considered as fingerprints of the spot are evidenced in both figures. In order to obtain a comparison of the currents generated over protein spots originating from different concentrations, a current profile curve was recorded while the tip was traveling over two spots at the same concentration corresponding to $0.1 \mathrm{ng}$ (1.7 nM BSA solution) of protein deposited on the surface but with different size. As was already visible in Fig. $2 b$, the current density varies over the spots due to some concentration effect that probably occurs during the evaporation steps in the preparation of the samples. As can be seen from Fig. 3, the $0.1 \mathrm{ng}$ spots are clearly
Table. Current intensity recorded over the $0.1 \mathrm{ng}$ spots, as well as background current values far from the spots.

\begin{tabular}{|lllll}
$\begin{array}{l}\text { Spot } \\
\text { (protein amount) }\end{array}$ & $\begin{array}{l}\text { Peak current } \\
\text { [Ampere] }\end{array}$ & $\begin{array}{l}\text { Average noise } \\
\text { [Ampere] }\end{array}$ & $\begin{array}{l}\text { Standard } \\
\text { deviation of the } \\
\text { noise } \\
\text { [Ampere] }\end{array}$ & $\begin{array}{l}\text { Theoretical limit } \\
\text { of detection } \\
\text { [Ampere] }\end{array}$ \\
\hline $0.1 \mathrm{ng}$ & $-1.0 \mathrm{E}-8$ & $-6.0 \mathrm{E}-10$ & $3.4 \mathrm{E}-10$ & $-1.7 \mathrm{E}-9$
\end{tabular}

Peak current values for two protein spots and statistical treatment of the data.

Current profile over two spots of protein (both at same concentration $1.5 \mathrm{nM}$ ) stained with copper. Scanning speed in X direction: $300 \mathrm{~mm} / \mathrm{s}, 25 \mathrm{~mm}$ platinum disk working electrode, silver wire as quasi reference electrode, platinum wire as a counter electrode. Equilibration time before scan: $50 \mathrm{~s}$ at $0.35 \mathrm{~V}$ vs Ag. $1 \mathrm{mM}$ ferrocene methanol aqueous solution, $0.1 \mathrm{M} \mathrm{KNO}_{3}$ as supporting electrolyte. $\mathrm{T}=25^{\circ} \mathrm{C}$

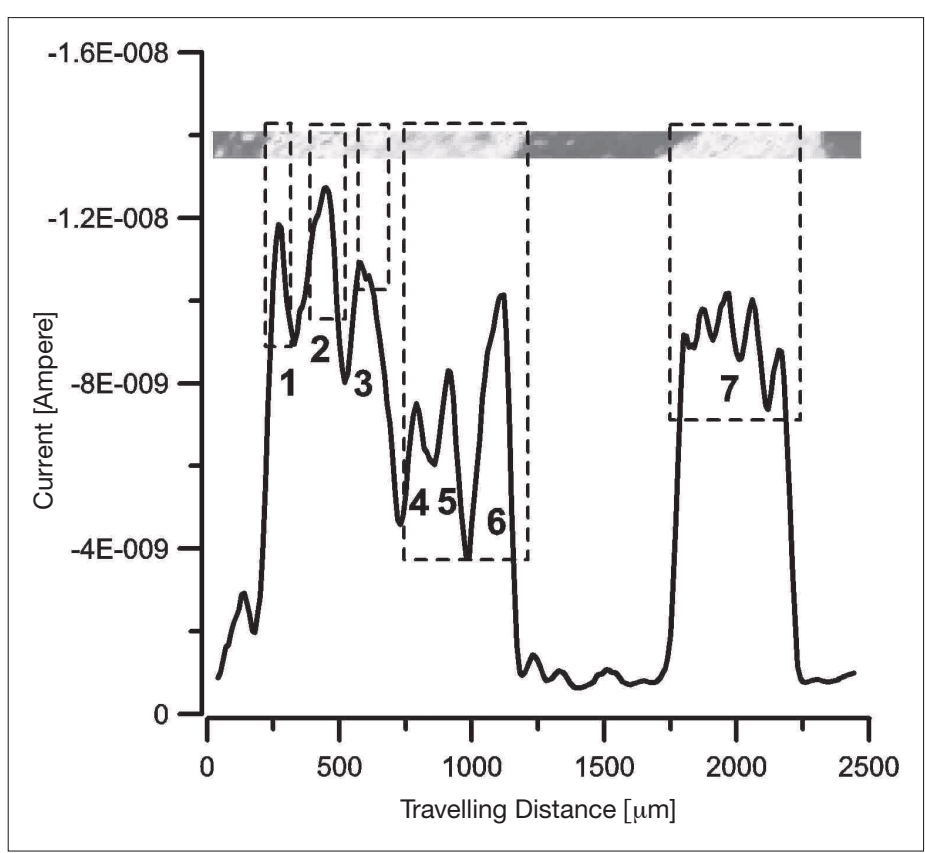

Fig. 3. Current profile over two spots of protein (same initial protein solution concentration but different size of the spots) stained with copper and then reduced. All conditions as in Fig. 1. The figure correlates the SECM image (upper part of the figure) to the current profile of a single scan along the $X$ direction (in both cases scan rate was $300 \mu \mathrm{m} / \mathrm{s}$ ). 
detectable over the background. If we compare the SECM scan (top part of the figure) with the linear scan along the $\mathrm{X}$ direction we can clearly notice the correspondence of the morphology. Each peak in the bottom part of the graph can be associated to each area of higher current densities in the SECM scan. Also current intensities in both plots match and this is important for the possibility of speeding up the detection process of stained proteins in membranes by decreasing the number of line scans. Complementarily, current intensities were measured over and between the spots, as presented in the Table. In particular, the background current far from the spots was measured. This allows estimation of the theoretical limit of detection of the system as (noise $+3 \times \sigma$ ) where 'noise' is the background level due to the oxidation of the mediator far from the spots, and $\sigma$ the standard deviation. The calculated theoretical limit of detection of $1.67 \times 10^{-9} \mathrm{~A}$ is roughly speaking an order of magnitude lower than the average signal obtained over the protein spots. This means that $0.1 \mathrm{ng}$ over $c a .1 \mathrm{~mm}^{2}$ is still above the practical limit of detection of the system. Very interestingly, this limit of detection is below the range of classical staining techniques used in membrane blotting analysis [1], (typically in the sub-nanogram range).

Whereas no true quantization possibility can be claimed from the above-described results, it must be stated that quantification is not the primary goal of this study, where emphasis is put on detectability. The important feature of protein spots detection systems in gel or electroblotted membrane analysis is not their ability to quantify proteins, but to detect low abundant proteins. The system presented above already reaches detection limits at least comparable to stateof-the-art techniques such as Coomassie or silver staining. SECM has thus the potential to surpass any classical staining technique in terms of sensitivity for the detection of protein spots on membranes. A possible development would be to show the ability to completely dissolve the deposited metal (whether by the SECM tip itself or more ad- vantageously chemically) to make possible protein recovery and subsequent analysis, typically by mass spectrometry.

We used herein a fast scanning speed of $300 \mu \mathrm{m} / \mathrm{s}$ to ensure the compatibility of the system with large surface (or long line) scanning, such as those encountered in gel electrophoresis followed by membrane electroblotting. However, scanning a $5 \times 5 \mathrm{~cm}$ area with a $100 \mu \mathrm{m}$ step between each scanning line would then require 23 h. This technique would be compatible as a whole surface scanning system only with miniaturized separations or with multi-tip scanning instruments. Alternatively, it can be envisioned as a technique complementary to classical stains to mine deeper in zones where protein spots are suspected but cannot be seen or to obtain fast scans along lanes of 1D gels. At the same time, the high speed used here, combined with a relatively large tip diameter of $25 \mu \mathrm{m}$, resulted in relatively low spatial resolution (pixel size is around $10 \mu \mathrm{m}$ in our scans). But SECM can also be pushed towards high resolution imaging: Bard et al. [11] calculated that under optimized conditions (substrate-tip distance, tip diameter...), detection of features 10 to 20 times smaller that the tip diameter should be feasible. This would also be useful in the detection of low abundant proteins on pseudo two-dimensional surfaces.

\section{Conclusions}

A new approach to the detection of low abundant proteins on PVDF membranes was presented: proteins are first stained with copper sulphate, copper is then reduced to give the metal and the surface is then scanned by the SECM tip, with ferrocene methanol acting as an electrochemical mediator to detect copper dissolution. The initial experimental results presented herein show that in this scheme, SECM can detect amounts of proteins as low as $0.1 \mathrm{ng}$ (over an area of ca. $1 \mathrm{~mm}^{2}$ ) on PVDF membranes, which is already comparable to state-of-the-art staining techniques. However, the potential for improvement in terms of detection sensitivity is so huge that despite its relatively low scanning speed, SECM should prove useful in routine analytical biochemistry.

\section{Acknowledgements}

The authors are part of the TMR network SUSANA (Supramolecular Self Assembly of Interfacial Nanostructures).

Received: December 17, 2004

[1] S. Harper, D.W. Speicher, in 'Current Protocols in Protein Science', Eds. J.E. Coligan, B.M. Dunn, H.L. Ploegh, D.W. Speicher, P.T. Wingfield, John Wiley \& Sons, New York, USA, 1995, Unit 10.8.

[2] J. Wen, M. Zhang, T.P. Horan, T. Li, J. Wypych, E.A. Mendiaz, K.E. Langley, K.H. Aoki, M. Kuwamoto, J.S. Philo, Y. Kita, T. Arakawa, Biosci., Biotechnol. and Biochem. 2001, 65, 1315-1320.

[3] M. Moeremans, G. Daneels, J. Demey, Anal. Biochem. 1985, 145, 315.

[4] C. Lee, A. Levin, D. Branton, Anal. Biochem. 1987, 166, 308-312.

[5] A.J. Bard, M.V. Mirkin, 'Scanning Electrochemical Microscopy', Marcel Dekker, New York, USA, 2001

[6] D. Mandler, A.J. Bard, J. Electrochem. Soc. 1989, 136, 3143.

[7] A.J. Bard, F.R. Fan, M.V. Mirkin, in 'Electroanalytical Chemistry', Eds. A.J. Bard, M.V. Mirkin, Marcel Dekker, New York, USA, 1994, 243.

[8] Biorad, http://wwww.biorad.com, 2004.

[9] W.J. Miao, Z.F. Ding, A.J. Bard, J. Phys. Chem. B 2002, 106, 1392-1398.

[10] A.J. Bard, L.R. Faulkner, 'Electrochemical Methods', Wiley \& Sons, New York, USA, 2001.

[11] A.J. Bard, M.V. Mirkin, P.R. Unwin, D.O. Wipf, J. Phys. Chem. 1992, 96, 18611868. 